\title{
Singularities of non-developable ruled surface with spacelike ruling
}

\author{
R. A. Abdel-Baky* and M. Khalifa Saad ${ }^{\dagger}$ \\ ${ }^{\star}$ Dept. of Math., Sciences Faculty for Girls, Jeddah University, 21352 Jeddah, KSA \\ ${ }^{\dagger}$ Dept. of Math., Faculty of Science, Islamic University of Madinah, 170 Al-Madinah, KSA \\ ${ }^{\star}$ Dept. of Math., Faculty of Science, Assiut University, 71516 Assiut, EGYPT \\ ${ }^{\dagger}$ Dept. of Math., Faculty of Science, Sohag University, 82524 Sohag, EGYPT
}

\begin{abstract}
In this paper, we study singularities of the spherical indicatrix and evolute of spacelike ruled surface with spacelike ruling. Finally, we give an example to illustrate our results.
\end{abstract}

Keywords. Blaschke frame, evolute of the dual spherical curve, singularity.

MSC (2000). 53A25, 53A05, 58A05.

\section{Introduction}

In the space, a continuously moving of a straight line generates a surface which is called ruled surface. Ruled surfaces have the most important positions and applications in the study of design problems in spatial mechanisms and physics, kinematics and computer aided design (CAD).So, these surfaces are one of the most important topics of surface theory. Because of this position of ruled surfaces, many geometers and engineers have investigated and obtained many properties of the ruled surfaces. There exists a vast literature on the subject including several monographs (See for instance $\left[\begin{array}{ll}1 & 5\end{array}\right]$ ). Furthermore, the differential geometry of the ruled surfaces in Minkowski space $\mathbb{E}_{1}^{3}$ is much more complicated than the Euclidean case, since Lorentzian metric is not positive definite metric, the distance function $<,>$ can be positive, negative or zero, whereas the distance function in the Euclidean space can only be positive. For instance, a continuously moving of a timelike line along a curve generates a timelike ruled surface. Turgut and Hacisalihoğlu have studied timelike ruled surfaces in Minkowski 3-space and given some properties of these surfaces [6]. Timelike ruled surface with timelike rulings have been studied by Abdel-All and others [7]. Küçük has obtained some results on the developable timelike ruled surfaces in the same space [8]. Furthermore, Uğurlu and Onder have introduced Frenet frames and Frenet invariants of timelike ruled surfaces in Minkowski 3-space [9].

One of the main techniques for applying the singularity theory to Euclidean differential geometry

\footnotetext{
* E-mail address: rbaky@Live.com

${ }^{\dagger}$ E-mail address: mohamed_khalifa77@science.sohag.edu.eg
} 
is to consider the distance squared function and the height function on a submanifold of $\mathbb{E}^{3}[10$. 12 . There are some articles concerning singularities of surfaces and classical geometric invariants of space curves for several kinds of geometry [13. In these articles the corresponding functions depend on each geometry. This work is dealing with the geometric analysis and the singularity of the spherical indicatrix and evolute of spacelike ruled surface with spacelike ruling Finally, an example illustrates the application of the obtained formulae was introduced.

\section{Basic concepts}

To meet the requirements in the next sections, here, the basic elements of the theory of curves in the Minkowski 3-space $\mathbb{E}_{1}^{3}$ are briefly presented. There exists a vast literature on the subject including several monographs, see for example [4,5].

Let $\mathbb{E}_{1}^{3}$ be the three-dimensional Minkowski space, that is, the three-dimensional real vector space $\mathbb{R}^{3}$ with the metric

$$
<d \mathbf{x}, d \mathbf{x}>=-d x_{1}^{2}+d x_{2}^{2}+d x_{3}^{2}
$$

where $\left(x_{1}, x_{2}, x_{3}\right)$ denotes the canonical coordinates in $\mathbb{R}^{3}$. An arbitrary vector $\mathbf{x}$ of $\mathbb{E}_{1}^{3}$ is said to be spacelike if $\langle\mathbf{x}, \mathbf{x}>>0$ or $\mathbf{x}=\mathbf{0}$, timelike if $\langle\mathbf{x}, \mathbf{x}><0$ and lightlike or null if $<\mathbf{x}, \mathbf{x}>=0$ and $\mathbf{x} \neq \mathbf{0}$. A timelike or light-like vector in $\mathbb{E}_{1}^{3}$ is said to be causal. For $\mathbf{x} \in \mathbb{E}_{1}^{3}$ the norm is defined by $\|\mathbf{x}\|=\sqrt{|<\mathbf{x}, \mathbf{x}>|}$, then the vector $\mathbf{x}$ is called a spacelike unit vector if $\langle\mathbf{x}, \mathbf{x}\rangle=1$ and a timelike unit vector if $\langle\mathbf{x}, \mathbf{x}\rangle=-1$. Similarly, a regular curve in $\mathbb{E}_{1}^{3}$ can locally be spacelike, timelike or null (lightlike), if all of its velocity vectors are spacelike, timelike or null (lightlike), respectively. For any two vectors $\mathbf{x}=\left(x_{1}, x_{2}, x_{3}\right)$ and $\mathbf{y}=\left(y_{1}, y_{2}, y_{3}\right)$ of $\mathbb{E}_{1}^{3}$, the inner product is the real number $<\mathbf{x}, \mathbf{y}>=-x_{1} y_{1}+x_{2} y_{2}+x_{3} y_{3}$ and the vector product is defined by

$$
\mathbf{x} \times \mathbf{y}=\left|\begin{array}{ccc}
-\mathbf{e}_{1} & \mathbf{e}_{2} & \mathbf{e}_{3} \\
x_{1} & x_{2} & x_{3} \\
y_{1} & y_{2} & y_{3}
\end{array}\right|=\left(-\left(x_{2} y_{3}-x_{3} y_{2}\right),\left(x_{3} y_{1}-x_{1} y_{3}\right),\left(x_{1} y_{2}-x_{2} y_{1}\right)\right)
$$

The hyperbolic and Lorentzian (de Sitter space) unit spheres are:

$$
\mathbb{H}_{+}^{2}=\left\{\mathbf{x} \in \mathbb{E}_{1}^{3} \mid-x_{1}^{2}+x_{2}^{2}+x_{3}^{2}=-1\right\}
$$

and

$$
\mathbb{S}_{1}^{2}=\left\{\mathbf{x} \in \mathbb{E}_{1}^{3} \mid-x_{1}^{2}+x_{2}^{2}+x_{3}^{2}=1\right\}
$$

respectively.

Definition 1 Let us consider two non-null vectors $\mathbf{x}$ and $\mathbf{y}$ in $\mathbb{E}_{1}^{3}$.

i) Let us assume that $\mathbf{x}$ and $\mathbf{y}$ are spacelike vectors, then

- If they span a spacelike plane; there is s a unique real number $0 \leq \theta \leq \pi$ such that $<\mathbf{x}, \mathbf{y}>=$ $\|\mathbf{x}\|\|\mathbf{y}\| \cos \theta$. This number is called the spacelike angle between the vectors $\mathbf{x}$ and $\mathbf{y}$. 
- If they span a timelike plane; there is s a unique real number $\theta \geq 0$ such that $\langle\mathbf{x}, \mathbf{y}\rangle=$ $\epsilon\|\mathbf{x}\|\|\mathbf{y}\| \cosh \theta$, where $\epsilon=+1$ or $\epsilon=-1$ according to $\operatorname{sign}\left(x_{2}\right)=\operatorname{sign}\left(y_{2}\right)$ or $\operatorname{sign}\left(x_{2}\right) \neq \operatorname{sign}\left(y_{2}\right)$, respectively. This number is called the central angle between the vectors $\mathbf{x}$ and $\mathbf{y}$.

ii) Let us assume that $\mathbf{x}$ and $\mathbf{y}$ are timelike vectors, then there is a unique real number $\theta \geq 0$ such that $\langle\mathbf{x}, \mathbf{y}\rangle=\epsilon\|\mathbf{x}\|\|\mathbf{y}\| \cosh \theta$, where $\epsilon=+1$ or $\epsilon=-1$ according to $\mathbf{x}$ and $\mathbf{y}$ have different timeorientation or the same time-orientation, respectively. This number is called the Lorentzian timelike angle between the vectors $\mathbf{x}$ and $\mathbf{y}$.

iii) Let us assume that $\mathbf{x}$ is spacelike and $\mathbf{y}$ is timelike, then there is a unique real number $\theta \geq 0$ such that $\langle\mathbf{x}, \mathbf{y}\rangle=\epsilon\|\mathbf{x}\|\|\mathbf{y}\| \sinh \theta$, where $\epsilon=+1$ or $\epsilon=-1$ according to $\operatorname{sign}\left(x_{2}\right)=\operatorname{sign}\left(y_{1}\right)$ or $\operatorname{sign}\left(x_{2}\right) \neq \operatorname{sign}\left(y_{1}\right)$. This number is called the Lorentzian timelike angle between the vectors $\mathbf{x}$ and $\mathbf{y}$.

Let $\alpha=\alpha(s)$ be a unit speed non-null curve in $\mathbb{E}_{1}^{3}$ has a spacelike or timelike rectifying plane; by $\kappa(s)$ and $\tau(s)$ we denote the natural curvature and torsion of $\alpha=\alpha(s)$, respectively. Then $\alpha(s)$ is called Serret-Frenet curve if $\kappa>0$, and $\tau \neq 0$. Consider the Serret-Frenet frame $\{\mathbf{t}(s), \mathbf{n}(s), \mathbf{b}(s)\}$ associated with the curve $\alpha=\alpha(s)$, then the Serret-Frenet formulae read as follows [7-9]:

$$
\left(\begin{array}{l}
\mathbf{t}^{\prime} \\
\mathbf{n}^{\prime} \\
\mathbf{b}^{\prime}
\end{array}\right)=\left(\begin{array}{lll}
0 & \kappa & 0 \\
-\epsilon_{0} \epsilon_{1} \kappa & 0 & \tau \\
0 & -\epsilon_{1} \epsilon_{2} \tau & 0
\end{array}\right)\left(\begin{array}{l}
\mathbf{t} \\
\mathbf{n} \\
\mathbf{b}
\end{array}\right)
$$

where $\epsilon_{0}=<\mathbf{t}, \mathbf{t}>= \pm 1, \epsilon_{1}=<\mathbf{n}, \mathbf{n}>= \pm 1, \epsilon_{2}=<\mathbf{b}, \mathbf{b}>= \pm 1, \epsilon_{0} \epsilon_{1} \epsilon_{2}=-1$. Here "prime" denotes the derivative with respect to the arc length parameter $s$. Now on the base of the different values of $\epsilon_{0}, \epsilon_{1}$, and $\epsilon_{2}$, we provide three detailed classifications for $\alpha=\alpha(s)$ :

(1) $\alpha=\alpha(s)$ is timelike curve if $-\epsilon_{0}=\epsilon_{1}=\epsilon_{2}=1$,

(2) $\alpha=\alpha(s)$ is called the first kind of spacelike curve $\epsilon_{0}=-\epsilon_{1}=\epsilon_{2}=1$,

(3) $\alpha=\alpha(s)$ is called the secon kind of spacelike curve $\epsilon_{0}=\epsilon_{1}=-\epsilon_{2}=1$.

Furthermore, if $\alpha(s)$ is a spherical curve in $\mathbb{S}_{1}^{2}$, by a translation in $\mathbb{E}_{1}^{3}$ if necessary, we may assume that

$$
<\alpha(s), \alpha(\mathbf{s})>=a,
$$

where $a$ is a constant. Without loss of generality we may assume that $a=1$. Then, we define a unit vector

$$
\mathbf{g}(s)=\alpha \times \mathbf{t} .
$$

It is easy to see that $\alpha(s), \mathbf{t}(s)$ and $\mathbf{g}(s)$ form an orthonormal basis along the curve $\alpha(s) \in \mathbb{S}_{1}^{2}$. Here, it is convenient to assume $\langle\alpha, \alpha>=1,<\mathbf{t}, \mathbf{t}\rangle=-1$, and $<\mathbf{g}, \mathbf{g}>=1$. Hence, Eq. (4) yields

$$
\alpha \times \mathbf{g}=\mathbf{t}, \quad \mathbf{t} \times \mathbf{g}=\alpha, \alpha \times \mathbf{t}=\mathbf{g} .
$$

Since $\alpha(s), \mathbf{t}(s)$ and $\mathbf{g}(s)$ form an orthonormal basis along the curve $\alpha(s) \in \mathbb{S}_{1}^{2}$, we call $\{\alpha(s), \mathbf{t}(s)$ $\mathbf{g}(s)\}$ the spherical Blaschke frame of the spherical curve $\alpha(s) \in \mathbb{S}_{1}^{2}$. By a direct computation, we 
conclude that there exists a function $\gamma(s)$ satisfying that

$$
\left(\begin{array}{l}
\alpha^{\prime} \\
\mathbf{t}^{\prime} \\
\mathbf{g}^{\prime}
\end{array}\right)=\left(\begin{array}{lll}
0 & 1 & 0 \\
1 & 0 & \gamma \\
0 & \gamma & 0
\end{array}\right)\left(\begin{array}{l}
\alpha \\
\mathbf{t} \\
\mathbf{g}
\end{array}\right) .
$$

Moreover, we call $\gamma(s)$ the spherical (geodesic) curvature function of the spherical curve $\alpha(s) \in \mathbb{S}_{1}^{2}$. It can be shown that

$$
\mathbf{b}(s)=\frac{\alpha^{\prime} \times \alpha^{\prime \prime}}{\left\|\alpha^{\prime} \times \alpha^{\prime \prime}\right\|}=\frac{\gamma \alpha-\mathbf{g}}{\sqrt{1+\gamma^{2}}} .
$$

It is obvious that $\mathbf{b}$ is the curvature axis or evolute of the spherical curve $\alpha(s) \in \mathbb{S}_{1}^{2}$. In fact, it is important to consider the relations between the spherical curvature $\gamma(\mathrm{s})$ and the curvature $\kappa(\mathrm{s})$, the torsion $\tau(\mathrm{s})$ of $\alpha(s) \in \mathbb{S}_{1}^{2}$. Therefore, from Eqs. (3) and (6) we have

$$
\kappa \mathbf{n}=\mathbf{t}^{\prime}=\alpha+\gamma \mathbf{g}
$$

By computing the inner products with both sides of the above equation respectively, we obtain

$$
\kappa^{2}=1+\gamma^{2}
$$

Differentiating both sides of Eq. (8), it is direct to generate

$$
\kappa \kappa^{\prime}=\gamma \gamma^{\prime}
$$

Differentiating both sides of Eq. (7) and using formulas (3)-(6), it follows that

$$
\kappa^{2} \mathbf{t}+\kappa^{\prime} \mathbf{n}+\kappa \tau \mathbf{b}=\left(1+\gamma^{2}\right) \mathbf{t}+\gamma^{\prime} \mathbf{g}
$$

Since $<\mathbf{t}, \mathbf{g}>=<\mathbf{t}, \alpha>=<\mathbf{t}, \mathbf{n}>=0$, the above equation gives

$$
\kappa^{2}=1+\gamma^{2}
$$

Similarly, by computing the inner products with both sides of Eq. (10), we get

$$
\kappa^{\prime 2}+\kappa^{2} \tau^{2}=\gamma^{\prime 2}
$$

Then Eqs. (9), (12) and (13) lead to

$$
\tau= \pm \frac{\gamma^{\prime}}{1+\gamma^{2}}
$$

Therefore, combining of Eqs. (12) and (14) generates the following relationships:

$$
\left.\begin{array}{c}
\kappa(s)=\frac{\left\|\alpha^{\prime} \times \alpha^{\prime \prime}\right\|}{\left\|\alpha^{\prime}\right\|^{3}}=\sqrt{1+\gamma^{2}}, \\
\tau(s)=\frac{\operatorname{det}\left(\alpha^{\prime}, \alpha^{\prime \prime}, \alpha^{\prime \prime \prime}\right)}{\left\|\alpha^{\prime} \times \alpha^{\prime \prime}\right\|^{2}}= \pm \frac{\gamma^{\prime}}{1+\gamma^{2}} .
\end{array}\right\}
$$


On the other hand, let us consider a circle on $\mathbb{S}_{1}^{2}$ is described by the equation

$$
\mathbb{S}_{1}^{1}\left(c, \mathbf{b}_{0}\right)=\left\{\alpha \in \mathbb{S}_{1}^{2} \mid<\alpha, \mathbf{b}_{0}>=c\right\}
$$

where $c$ is a real constant, and $\mathbf{b}_{0}$ is a fixed spacelike unit vector which determines the circle's center. $\mathbb{S}_{1}^{1}\left(c, \mathbf{b}_{0}\right)$ This means that $\mathbb{S}_{1}^{1}\left(c, \mathbf{b}_{0}\right)$ is a great circle if $c=0$, and a small circle if $c \neq 0$, respectively. Then, we have:

Proposition 1. Let $\alpha: I \subseteq \mathbb{R} \rightarrow \mathbb{S}_{1}^{2}$ be a unit speed timelike curve. Then $\alpha(s)$ is a part of $\mathbb{S}_{1}^{1}\left(0, \mathbf{b}_{0}\right)$ if and only if $\gamma(s)=0$.

Proof. Suppose that $\gamma(s)=0$. By the Blaschke formula (6), we have $\mathbf{g}$ is a constant unit vector. We consider a function $f: I \subseteq \mathbb{R} \rightarrow \mathbb{R}$ defined by $f(s)=<\mathbf{x}, \mathbf{b}_{0}>$. Then we have $f(s)=\left\langle\mathbf{x}, \mathbf{b}_{0}>=0\right.$, and $f^{\prime}=<\mathbf{t}, \mathbf{b}_{0}>=0$. Therefore $f$ is identically equal to 0 , and so $\mathbf{x}(s) \subset \mathbb{S}_{1}^{1}\left(0, \mathbf{b}_{0}\right)$.

Conversely, assume that there exists $\mathbb{S}_{1}^{1}\left(0, \mathbf{b}_{0}\right)$ such that $\mathbf{x}(s) \subset \mathbb{S}_{1}^{1}\left(0, \mathbf{b}_{0}\right)$ Then the function $f$ as above is identically equal to zero. Ifollows that $f^{\prime}=<\mathbf{t}, \mathbf{b}_{0}>=0$. Thus

$$
f^{\prime \prime}=<\mathbf{t}^{\prime}, \mathbf{b}_{0}>=<\alpha+\gamma \mathbf{g}, \mathbf{b}_{0}>=\gamma<\mathbf{g}, \mathbf{b}_{0}>=0 .
$$

Since $\gamma \neq 0$, we have $<\mathbf{g}, \mathbf{b}_{0}>=0$. It follows that $<\mathbf{g}^{\prime}, \mathbf{b}_{0}>=\gamma<\mathbf{t}, \mathbf{b}_{0}>=0$. Since $\gamma \neq 0$, we have $<\mathbf{g}, \mathbf{b}_{0}>=<\mathbf{t}, \mathbf{b}_{0}>=0$. Then $\mathbf{b}_{0}= \pm \mathbf{t} \times \mathbf{g}$ which contradicts to the fact that $\mathbf{t} \times \mathbf{g}=\alpha$. Thus $\gamma=0$ for any $s \in I$

Corollary 1. Let $\alpha: I \subseteq \mathbb{R} \rightarrow \mathbb{S}_{1}^{2}$ be a unit speed timelike curve. Then $\gamma^{\prime}=0$ if and only if $\alpha(s)$ is a part of $\mathbb{S}_{1}^{1}\left(c, \mathbf{b}_{0}\right) \subset \mathbb{S}_{1}^{2}$.

Proof. For the first differential of $\mathbf{b}$ we get:

$$
\mathbf{b}^{\prime}=\mp \frac{\gamma^{\prime}}{\gamma^{2}+1}\left(\frac{\alpha-\gamma \mathbf{g}}{\sqrt{\gamma^{2}+1}}\right) \text {. }
$$

Then $\mathbf{b}_{0}= \pm \mathbf{b}$ if and only if $\gamma^{\prime}=0$. From Eqs. (14) we can figure out that $\gamma^{\prime}=0$ yields that $\tau=0$ and $\kappa$ is constant directly, which implies that $\alpha(s) \in \mathbb{S}_{1}^{2}$ is a part of the small circle $\mathbb{S}_{1}^{1}\left(c, \mathbf{b}_{0}\right)$ whose center is $\mathbf{b}_{0}$.

\section{Spacelike ruled surface with spacelike ruling}

A spacelike ruled surface in Minkowski 3 -space $\mathbb{E}_{1}^{3}$ can be written as:

$$
M: \mathbf{y}(s, v)=\mathbf{c}(s)+v \mathbf{x}(s), s \in I, v \in \mathbb{R},
$$

with

$$
<\mathbf{x}, \mathbf{x}>=1,<\mathbf{c}^{\prime}, \mathbf{x}^{\prime}>=0
$$

Here $\mathbf{c}(s)$ is a spacelike curve and $\mathbf{x}$ is unit vector moving a long $\mathbf{c}(s)$. In this case the curve $\mathbf{c}=\mathbf{c}(s)$ is the striction curve, and the parameter $s$ is the arc length of spherical image or indicatrix $\mathbf{x}(s) \in \mathbb{S}_{1}^{2}$. 
Generally, we call such an expression the standard equation of the non-developable spacelike ruled surfaces with spacelike ruling in Minkowski 3-space $\mathbb{E}_{1}^{3}$. Here we will take the notation $\mathbf{x}(s)=\alpha(s)$. Since $\left\langle\mathbf{c}^{\prime}, \mathbf{x}^{\prime}\right\rangle=0$, it is reasonable to assume

$$
\mathbf{c}^{\prime}=\Gamma \mathbf{x}+\mu \mathbf{g}
$$

The functions $\gamma(s), \Gamma(s)$ and $\mu(s)$ are called the curvature functions or construction parameters of the ruled surface. The geometrical meanings of these invariants are explained as follows: $\gamma$ is the geodesic curvature of the spherical image curve $\mathbf{x}=\mathbf{x}(s) ; \Gamma$ describes the angle between the tangent of the striction curve and the ruling of the surface; and $\mu$ is the distribution parameter of the ruled surface $M$ at the ruling $\mathbf{x}$.

\subsection{Lorentzian height functions}

In this subsection we introduce a family of functions which is useful for the study of geometric invariants of timelike spherical curve $\mathbf{x}(s)$ in $\mathbb{S}_{1}^{2}$. For this purpose, similar to the books in [10,11], the spacelike fixed unit vector $\mathbf{b}_{0}$ of $\mathbb{S}_{1}^{2}$ will be said to be a $\mathbf{b}_{k}$ evolute of the timelike curve $\mathbf{x}(s)$ in $\mathbb{S}_{1}^{2}$ at $s \in I$ if for all $i$ such that $1 \leq i \leq k,<\mathbf{b}_{0}, \mathbf{x}^{i}(s)>=0$, but $\left\langle\mathbf{b}_{0}, \mathbf{x}^{k+1}(s)>\neq 0\right.$. Here $\mathbf{x}^{i}$ denotes the $\mathrm{i}$-th derivatives of $\mathbf{x}$ with respect to the arc length of $\mathbf{x}(s) \in \mathbb{S}_{1}^{2}$.

We now define a smooth function $H^{T}: I \times \mathbb{S}_{1}^{2} \rightarrow \mathbb{R}$, by $H^{T}\left(s, \mathbf{b}_{0}\right)=<\mathbf{b}_{0}, \mathbf{x}>$. We call $H^{T}$ a Lorentzian height function of $\mathbf{x}(s)$ in $\mathbb{S}_{1}^{2}$. We use the notation $h_{0}(s)=H^{T}\left(s, \mathbf{b}_{0}\right)$ for any fixed spacelike unit vector $\mathbf{b}_{0}$ of $\mathbb{S}_{1}^{2}$. Then we have the following proposition.

Proposition 2. Under the aforementioned notations, by direct calculation, the following holds: 1- $h_{0}$ will be invariant in the first approximation if and only if $\mathbf{b}_{0} \in S p\{\mathbf{x}, \mathbf{g}\}$, that is,

$$
h_{\mathbf{0}}^{\prime}=0 \Leftrightarrow<\mathbf{x}^{\prime}, \mathbf{b}_{0}>=0 \Leftrightarrow<\mathbf{t}, \mathbf{b}_{0}>=0 \Leftrightarrow \mathbf{b}_{0}=a_{1} \mathbf{x}+a_{2} \mathbf{g}
$$

for some numbers $a_{1}, a_{2} \in \mathbb{R}$, and $a_{1}^{2}+a_{2}^{2}=1$.

2- $h_{0}$ will be invariant in the second approximation if and only if $\mathbf{b}_{0}$ is $\mathbf{b}_{2}$ evolute of $\mathbf{x}(s) \in \mathbb{S}_{1}^{2}$, that is,

$$
h_{0}^{\prime}=h_{0}^{\prime \prime}=0 \Leftrightarrow \mathbf{b}_{0}= \pm \mathbf{b} .
$$

3- $h_{0}$ will be invariant in the third approximation if and only if $\mathbf{b}_{0}$ is $\mathbf{b}_{3}$ evolute of $\mathbf{x}(s) \in \mathbb{S}_{1}^{2}$, that is,

$$
h_{0}^{\prime}=h_{0}^{\prime \prime}=h_{0}^{\prime \prime \prime}=0 \Leftrightarrow \mathbf{b}_{0}= \pm \mathbf{b}, \text { and } \gamma^{\prime}=0 .
$$

4- $h_{0}$ will be invariant in the fourth approximation if and only if $\mathbf{b}_{0}$ is $\mathbf{b}_{4}$ evolute of $\mathbf{x}(s) \in \mathbb{S}_{1}^{2}$, that is,

$$
h_{0}^{\prime}=h_{0}^{\prime \prime}=h_{0}^{\prime \prime \prime}=h_{0}^{(4)}=0 \Leftrightarrow \mathbf{b}_{0}= \pm \mathbf{b}, \text { and } \gamma^{\prime}=\gamma^{\prime \prime}=0 .
$$

By Proposition 2, we now discuss the contact of $\mathbf{x}(s)$ with the circle $\mathbb{S}_{1}^{1}\left(c, \mathbf{b}_{0}\right)$ : (a). There exist a spacelike vector $\mathbf{b}_{0}=\mathbf{b}$ such that $\mathbf{x}(s) \subset \mathbb{S}_{1}^{1}\left(c, \mathbf{b}_{0}\right)$ if and only if $\gamma^{\prime}(s)=0$ In this case $\mathbb{S}_{1}^{1}\left(c, \mathbf{b}_{0}\right)$ has at least 3 -point contact with $\mathbf{x}(s)$ at $s_{0}(\mathrm{~b})$. The circle $\mathbb{S}_{1}^{1}\left(c, \mathbf{b}_{0}\right)$ and the curve $\mathbf{x}(s)$ in $\mathbb{S}_{1}^{2}$ have at least 4 -point contact at $\mathbf{x}\left(s_{0}\right)$ if and only if $\gamma^{\prime}=0$, and $\gamma^{\prime \prime} \neq 0$. 


\subsection{Unfoldings of functions of one variable}

In this section we will use the same technique on the singularity theory for families of smooth functions. Detailed descriptions are found in the books [11,12]. Let $F:\left(\mathbb{R} \times \mathbb{R}^{r},\left(s_{0}, \mathbf{x}_{0}\right)\right) \rightarrow \mathbb{R}$ be a smooth function, and $\mathcal{F}(s)=F_{x_{0}}, F_{x_{0}}(s)=F\left(s, \mathbf{x}_{0}\right)$. Then $F$ is called an r-parameter unfolding of $\mathcal{F}(s)$. If $\mathcal{F}^{(p)}\left(s_{0}\right)=0$ for all $1 \leq p \leq k+1$, and $\mathcal{F}^{(p+1)}\left(s_{0}\right) \neq 0$, we say $\mathcal{F}(s)$ has $A_{\leq k}$-singularity at $s_{0}$. We also say $\mathcal{F}(s)$ has $A_{\leq k}$-singularity at $s_{0}$ at if $\mathcal{F}^{(p)}\left(s_{0}\right)=0$ for all $1 \leq p \leq k+1$. Let $F$ be an r-parameter unfolding of $\mathcal{F}(s)$ and $\mathcal{F}(s)$ has $A_{k}$-singularity $(1 \leq k)$ at $s_{0}$, we define the $(k-1)$-jet of the partial derivative $\frac{\partial F}{\partial x_{i}}$ at $s_{0}$ be $j^{(k-1)}\left(\frac{\partial F}{\partial x_{i}}\left(s, \mathbf{x}_{0}\right)\right)=\gamma_{j=1}^{k-1} L_{j i}^{j} s$ (without the constant term), for $i=1, \ldots, r$. Then $F(s, \mathbf{x})$ is called a $(p)$ versal unfolding if and only if the $(k-1) \times r$ matrix of coefficients $\left(L_{j i}\right)$ has rank $(k-1)$. (This certainly requires $k-1 \leq r$, so the smallest value of $r$ is $k-1$ ).

We now state important sets about the unfoldings relative to the above notations. The singular set of $F(s, \mathbf{x})$ is the set

$$
\mathbb{S}_{F}=\left\{\mathbf{x} \in \mathbb{S}_{1}^{2} \mid \text { there exists } s \in \mathbb{R} \text { with } \frac{\partial F}{\partial s}=0 \text { at }(s, \mathbf{x})\right\} .
$$

The bifurcation set $\mathbb{B}_{F}$ of $F$ is the set $[12,13]$ :

$$
\mathbb{B}_{F}=\left\{\mathbf{x} \in \in \mathbb{S}_{1}^{2} \mid \text { there exists } s \in \mathbb{R} \text { with } \frac{\partial F}{\partial s}=\frac{\partial^{2} F}{\partial s^{2}}=0 \text { at }(s, \mathbf{x})\right\} .
$$

Then similar to [12], we can state the following theorem:

Theorem 1. Let $F:\left(\mathbb{R} \times \mathbb{R}^{r},\left(s_{0}, \mathbf{x}_{0}\right)\right) \rightarrow \mathbb{R}$ be an r-parameter unfolding of $\mathcal{F}(s)$, which has the $A_{k}$ singularity $(k \geq 1)$ at $s_{0}$. Suppose that $F$ is a $(p)$ versal unfolding Then:

(1) If $k=2$, then $\mathbb{B}_{F}$ is locally diffepmorphic to $\{\mathbf{0}\} \times \mathbb{R}^{r-1}$;

(2) If $k=3$, then $\mathbb{B}_{F}$ is locally diffepmorphic to $\mathbf{C} \times \mathbb{R}^{r-2}$, where $\mathbf{C}=\left\{\left(x_{1}, x_{2}\right) \mid x_{1}^{2}=x_{2}^{3}\right\}$ is the ordinary cusp.

For the given curve $\mathbf{x}(s) \in \mathbb{S}_{1}^{2}$, and $h_{\mathbf{0}}(s)=H^{T}\left(s, \mathbf{b}_{0}\right)$, the bifurcation set of $H^{T}$ is given as follows:

$$
\mathbb{B}_{H^{T}}=\left\{\mathbf{x} \in \mathbb{S}_{1}^{2} \mid \mathbf{b}= \pm \frac{\gamma \mathbf{x}-\mathbf{g}}{\sqrt{\gamma^{2}+1}} \cdot\right\}
$$

Hence, we have the following fundamental proposition:

Proposition 3. For the unit speed timelike curve $x(s)=\left(x_{1}(s), x_{2}(s), x_{3}(s)\right)$ on $\mathbb{S}_{1}^{2}$. If $h_{0}(s)=$ $H^{T}\left(s, \mathbf{b}_{0}\right)$ has the $A_{k}$-singularity $(k=2,3)$ at $s_{0} \in \mathbb{R}$, then $H^{T}$ is the $(p)$ versal unfolding of $h_{0}\left(s_{0}\right)$.

Proof. Let $b_{0}=\left(b_{1}, b_{2}, b_{3}\right) \in \mathbb{S}_{1}^{2}$, with $-b_{1}^{2}+b_{2}^{2}+b_{3}^{2}=1$ Then we have

$$
H^{T}\left(s, \mathbf{b}_{0}\right)=-b_{1} x_{1}(s)+b_{2} x_{2}(s)+b_{3} x_{3}(s) .
$$

Suppose that $b_{3}>0$, and $b_{3}=\sqrt{1+b_{1}^{2}-b_{2}^{2}}$. Then we have

$$
H^{T}\left(s, \mathbf{b}_{0}\right)=-b_{1} x_{1}(s)+b_{2} x_{2}(s)+\sqrt{1+b_{1}^{2}-b_{2}^{2}} x_{3}(s) .
$$


Therefore

$$
\left.\begin{array}{l}
\frac{\partial H^{T}}{\partial b_{1}}=\left(-x_{1}(s)+\frac{b_{1} x_{3}(s)}{\sqrt{1+b_{1}^{2}-b_{2}^{2}}}\right), \\
\frac{\partial H^{T}}{\partial b_{2}}=\left(x_{2}(s)-\frac{b_{2} x_{3}(s)}{\sqrt{1+b_{1}^{2}-b_{2}^{2}}}\right) .
\end{array}\right\}
$$

We also have

$$
\left.\begin{array}{rl}
\frac{\partial}{\partial s} \frac{\partial H^{T}}{\partial b_{1}} & =\left(-x_{1}^{\prime}(s)+\frac{b_{1} x_{3}^{\prime}(s)}{\sqrt{1+b_{1}^{2}-b_{2}^{2}}}\right), \\
\frac{\partial}{\partial s} \frac{\partial H^{T}}{\partial b_{2}} & =\left(x_{2}^{\prime}(s)-\frac{b_{2} x_{3}^{\prime}(s)}{\sqrt{1+b_{1}^{2}-b_{2}^{2}}}\right),
\end{array}\right\}
$$

and

$$
\left.\begin{array}{l}
\frac{\partial^{2}}{\partial s^{2}} \frac{\partial H^{T}}{\partial b_{1}}=\left(-x_{1}^{\prime \prime}(s)+\frac{b_{1} x_{3}^{\prime \prime}(s)}{\sqrt{1+b_{1}^{2}-b_{2}^{2}}}\right), \\
\frac{\partial^{2}}{\partial s^{2}} \frac{\partial H^{T}}{\partial b_{2}}=\left(x_{2}^{\prime \prime}(s)-\frac{b_{2} x_{3}^{\prime}(s)}{\sqrt{1+b_{1}^{2}-b_{2}^{2}}}\right) \cdot
\end{array}\right\}
$$

Thus

$$
\left.\begin{array}{l}
j^{1}\left(\frac{\partial H^{T}}{\partial b_{1}}\left(s, \mathbf{b}_{0}\right)\right)=\left(-x_{1}^{\prime}\left(s_{0}\right)+\frac{b_{1} x_{3}^{\prime}\left(s_{0}\right)}{b_{30}}\right) s, \\
j^{1}\left(\frac{\partial H^{T}}{\partial b_{2}}\left(s, \mathbf{b}_{0}\right)\right)=\left(x_{2}^{\prime}\left(s_{0}\right)-\frac{b_{2} x_{3}^{\prime}\left(s_{0}\right)}{b_{30}}\right) s,
\end{array}\right\}
$$

and

$$
\begin{gathered}
j^{2}\left(\frac{\partial H^{T}}{\partial b_{1}}\left(s, \mathbf{b}_{0}\right)\right)=\left(-x_{1}^{\prime}\left(s_{0}\right)+\frac{b_{1} x_{3}^{\prime}\left(s_{0}\right)}{b_{30}}\right) s \\
+\frac{1}{2}\left(-x_{1}^{\prime \prime}\left(s_{0}\right)+\frac{b_{1} x_{3}^{\prime \prime}\left(s_{0}\right)}{b_{30}}\right) s^{2} \\
j^{2}\left(\frac{\partial H^{T}}{\partial b_{2}}\left(s, \mathbf{b}_{0}\right)\right)=\left(x_{2}^{\prime}\left(s_{0}\right)-\frac{b_{2} x_{3}^{\prime}\left(s_{0}\right)}{b_{30}}\right) s \\
+\frac{1}{2}\left(x_{2}^{\prime \prime}\left(s_{0}\right)-\frac{b_{2} x_{3}^{\prime \prime}\left(s_{0}\right)}{b_{30}}\right) s^{2} .
\end{gathered}
$$

(i) If $h_{0}\left(s_{0}\right)$ has the $A_{2}$-singularity at $s_{0} \in \mathbb{R}$, then $h_{0}\left(s_{0}\right)=0$. So the $(2-1) \times 2$ matrix of coefficients $\left(L_{j i}\right)$ is:

$$
A=\left(-x_{1}^{\prime}\left(s_{0}\right)+\frac{b_{1} x_{3}^{\prime}\left(s_{0}\right)}{b_{30}} \quad x_{2}^{\prime}\left(s_{0}\right)-\frac{b_{2} x_{3}^{\prime}\left(s_{0}\right)}{b_{30}}\right) ;
$$

Suppose that the rank of the matrix $A$ is zero, then we have:

$$
x_{1}^{\prime}\left(s_{0}\right)=\frac{b_{1} x_{3}^{\prime}\left(s_{0}\right)}{b_{30}}, x_{2}^{\prime}\left(s_{0}\right)=\frac{b_{2} x_{3}^{\prime}\left(s_{0}\right)}{b_{30}} .
$$

Since $\left\|\mathbf{x}^{\prime}\left(s_{0}\right)\right\|^{2}=-1$, we have $x_{3}^{\prime}\left(s_{0}\right) \neq 0$, so that we have the contradiction as follows:

$$
\begin{aligned}
0 & =<\left(x_{1}^{\prime}\left(s_{0}\right), x_{2}^{\prime}\left(s_{0}\right), x_{3}^{\prime}\left(s_{0}\right)\right),\left(b_{1}, b_{2}, b_{30}\right)> \\
& =-x_{1}^{\prime}\left(s_{0}\right) b_{1}+x_{2}^{\prime}\left(s_{0}\right) b_{2}+x_{3}^{\prime}\left(s_{0}\right) b_{30} \\
& =-\frac{b_{1}^{2} x_{3}^{\prime}\left(s_{0}\right)}{b_{30}}+\frac{b_{2}^{2} x_{3}^{\prime}\left(s_{0}\right)}{b_{30}}+x_{3}^{\prime}\left(s_{0}\right) b_{30} \\
& =\frac{-x_{3}^{\prime}\left(s_{0}\right)}{b_{30}} \neq 0 .
\end{aligned}
$$


It means that rank $(A)=1$, and $H^{T}$ is the (p) versal unfolding of $h_{\mathbf{0}}$ at $s_{0}$.

(ii) If $h_{\mathbf{0}}\left(s_{0}\right)$ has the $A_{3}$-singularity at $s_{0} \in \mathbb{R}$, then $h_{0}^{\prime}=h_{0}^{\prime \prime}=0$, and by Proposition 1 :

$$
\mathbf{b}\left(s_{0}\right)= \pm\left(\frac{\gamma \mathbf{x}+\mathbf{g}}{\sqrt{\gamma^{2}+1}}\right)\left(s_{0}\right)
$$

where $\gamma^{\prime}\left(s_{0}\right)=0$, and $\gamma^{\prime \prime}\left(s_{0}\right) \neq 0$. So the $(3-1) \times 2$ matrix of the coefficients $\left(L_{j i}\right)$ is

$$
B=\left(\begin{array}{cc}
L_{11} & L_{12} \\
L_{21} & L_{22}
\end{array}\right)=\left(\begin{array}{cc}
-x_{1}^{\prime}+\frac{b_{1} x_{3}^{\prime}}{b_{30}} & x_{2}^{\prime}-\frac{b_{2} x_{3}^{\prime}}{\sqrt{1+b_{1}^{2}-b_{2}^{2}}} \\
-x_{1}^{\prime \prime}+\frac{b_{1} x_{3}^{\prime \prime}}{b_{30}} & x_{2}^{\prime \prime}-\frac{b_{2} x_{3}^{\prime}}{b_{30}}
\end{array}\right) .
$$

For the purpose, we also require the $2 \times 2$ matrix $B$ to be non-singular, which always does. In fact, the determinate of this matrix at $s_{0}$ is

$$
\begin{aligned}
\operatorname{det}(B) & =\frac{1}{b_{30}}\left|\begin{array}{ccc}
-x_{1}^{\prime} & x_{2}^{\prime} & x_{3}^{\prime} \\
-x_{1}^{\prime \prime} & x_{2}^{\prime \prime} & x_{3}^{\prime \prime} \\
b_{10} & b_{20} & b_{30}
\end{array}\right| \\
& =\frac{1}{b_{30}}<\mathbf{x}^{\prime} \times \mathbf{x}^{\prime \prime}, \mathbf{b}_{\mathbf{0}}> \\
& = \pm \frac{1}{b_{30}}<\mathbf{x}^{\prime} \times \mathbf{x}^{\prime \prime},\left(\frac{\gamma \mathbf{x}+\mathbf{g}}{\sqrt{\gamma^{2}+1}}\right)>
\end{aligned}
$$

Since $x^{\prime}=\mathbf{t}$, we have $x^{\prime \prime}=x+\gamma \mathbf{g}$. Substituting these relations to the above equality, we have:

$$
\operatorname{det}(B)=\mp \frac{\sqrt{\gamma^{2}\left(s_{0}\right)+1}}{b_{30}} \neq 0
$$

This means that rank $(B)=2$

Propostion 4. Let $x(s)$ be a unit speed timelike curve on $\mathbb{S}_{1}^{2}$, then the spherical evolute of $x(s)$ is:

(1) Diffepmorphic to a constant spacelike vector if $\gamma^{\prime}\left(s_{0}\right) \neq 0$;

(2) Diffepmorphic to the cusp $\mathbf{C}$ at $s_{0} \in \mathbb{R}$ if $\gamma^{\prime}\left(s_{0}\right)=0$, and $\gamma^{\prime \prime}\left(s_{0}\right) \neq 0$.

Proof. For the proof of assertion (1), from Eq. (22) we have:

$$
\mathbf{b}^{\prime}=\mp \frac{\gamma^{\prime}}{\left(\gamma^{2}+1\right)^{\frac{3}{2}}}(\mathbf{x}+\gamma \mathbf{g})
$$

Therefore $\mathbf{b}$ is locally diffepmorphic to a constant spacelike vector if $\gamma^{\prime}\left(s_{0}\right) \neq 0$. For the assertion (2), from Proposition 2, and Theorem 1, the bifurcation set $\mathbb{B}_{H^{T}}$ at $\mathbf{b}_{0}= \pm\left(\frac{\gamma \mathbf{x}-\mathbf{g}}{\sqrt{\gamma^{2}+1}}\right)\left(s_{0}\right)$ is locally diffepmorphic to the ordinary cusp $\mathbf{C}$ in $\mathbb{S}_{1}^{2}$ if $\gamma^{\prime}\left(s_{0}\right)=0$, and $\gamma^{\prime \prime}\left(s_{0}\right) \neq 0$

Example 1. Let a spacelike ruled defined by

$$
M: \mathbf{y}(u, v)=\mathbf{c}(u)+v \mathbf{x}(u), u \in I, v \in \mathbb{R},
$$


where

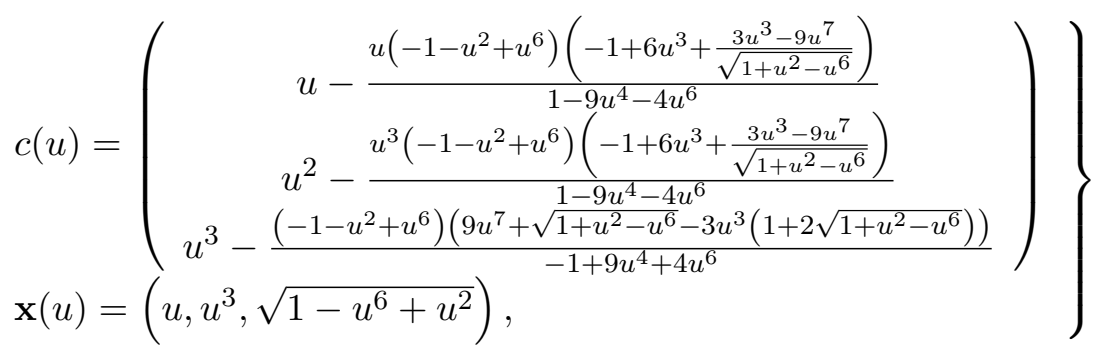

and $-0.55<u<0.55$. By a straightforward calculation, we have

$$
\left.\begin{array}{l}
<\mathbf{x}, \mathbf{x}>=1,<\mathbf{c}^{\prime}, \mathbf{x}^{\prime}>=0, \\
\mathbf{t}(u)=\left(1,3 u^{2}, \frac{2 u-6 u^{5}}{2 \sqrt{1+u^{2}-u^{6}}}\right), \\
\mathbf{g}(u)=\left(-\frac{u^{2}\left(3+2 u^{2}\right) \sqrt{-1-u^{2}+u^{6}}}{\sqrt{1-9 u^{4}-4 u^{6}} \sqrt{1+u^{2}-u^{6}}}, \frac{\sqrt{-1-u^{2}+u^{6}}\left(1+2 u^{6}\right)}{\sqrt{1-9 u^{4}-4 u^{6}} \sqrt{1+u^{2}-u^{6}}}, \frac{2 u^{3} \sqrt{-1-u^{2}+u^{6}}}{\sqrt{1-9 u^{4}-4 u^{6}}}\right) .
\end{array}\right\}
$$

Further, we get

$$
\begin{aligned}
\gamma(u) & =\frac{2 u \sqrt{-1-u^{2}+u^{6}}\left(-3-4 u^{2}+12 u^{6}+4 u^{8}\right)}{\left(1-9 u^{4}-4 u^{6}\right)^{3 / 2} \sqrt{1+u^{2}-u^{6}}}, \\
\gamma^{\prime}(u) & =-\frac{6 \sqrt{-1-u^{2}+u^{6}}\left(1+4 u^{2}+45 u^{4}+40 u^{6}+20 u^{8}+36 u^{10}+4 u^{12}\right)}{\left(1-9 u^{4}-4 u^{6}\right)^{5 / 2} \sqrt{1+u^{2}-u^{6}}} .
\end{aligned}
$$

It follows that the evolute $\mathbf{b}(u)$ is given as follows:

$$
\mathbf{b}(u)= \pm \frac{\gamma \mathbf{x}(u)-\mathbf{g}(u)}{\sqrt{\gamma^{2}+1}}=\frac{1}{\delta}\left(b_{1}(u), b_{2}(u), b_{3}(u)\right)
$$

where

$$
\begin{aligned}
& b_{1}(u)=-3 u^{2} \sqrt{-1-u^{2}+u^{6}}\left(1+2 u^{2}+9 u^{4}+2 u^{6}\right)\left(-1+9 u^{4}+4 u^{6}\right)^{3 / 2} \\
& b_{2}(u)=\sqrt{-1-u^{2}+u^{6}}\left(-1+9 u^{4}+4 u^{6}\right)^{3 / 2}\left(-1+3 u^{4}-6 u^{6}+42 u^{10}+16 u^{12}\right) \\
& b_{3}(u)=2 u \sqrt{-1-u^{2}+u^{6}}\left(-1+9 u^{4}+4 u^{6}\right)^{3 / 2}\left(-3-5 u^{2}+21 u^{6}+8 u^{8}\right) \text {, }
\end{aligned}
$$

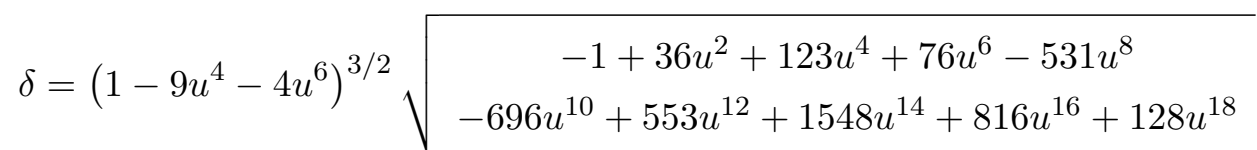

For the case that $u=$ ?, we have $\gamma^{\prime}(0)=0$, and $\gamma^{\prime \prime}(0) \neq 0$. According to Proposition 4 the osculating circle $\mathbb{S}_{1}^{1}\left(0, \mathbf{b}_{0}\right)$ has 3 -point contact with $\mathbf{x}(u)$ at $u=0$ and $\mathbf{b}(0)= \pm \mathbf{e}_{2}$. 


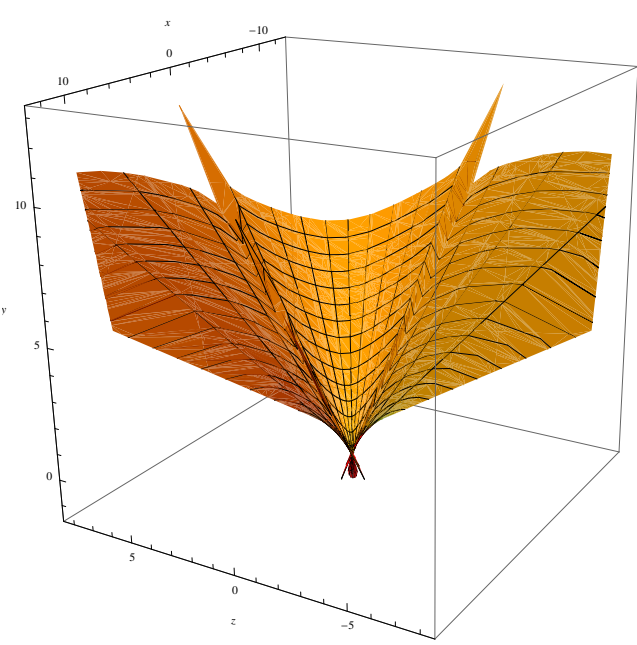

(a)

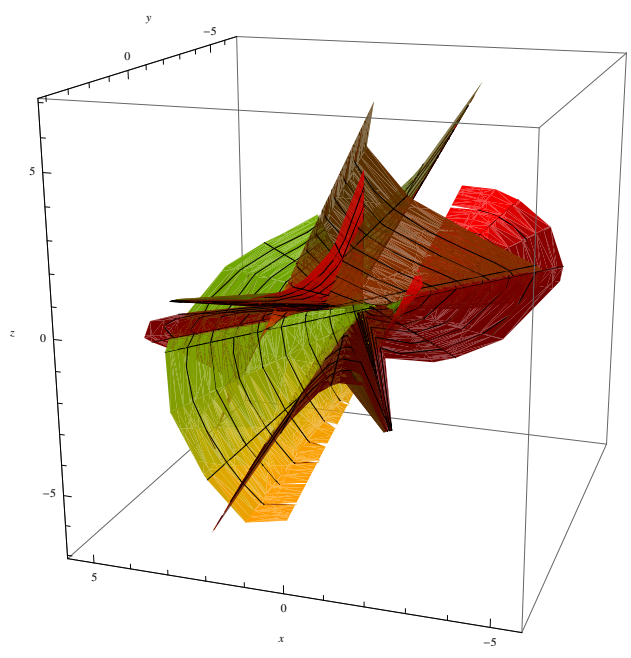

(b)

Figure 1: (a) The spacelike ruled surface $M$. (b) The evolute surface of $M$.

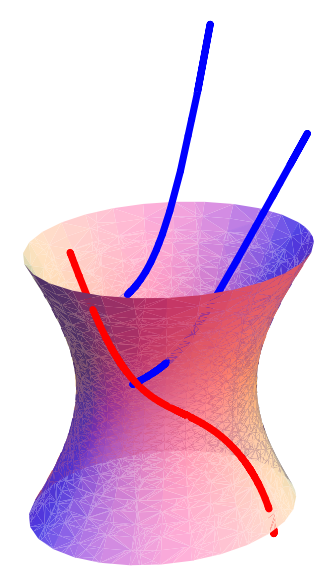

Figure 2: The space curve of the tangential surface.

\section{References}

[1] O. Bottema, and B. Roth. Theoretical Kinematics, North-Holland Press, New York 1979.

[2] A. Karger, and J. Novak. Space Kinematics and Lie Groups, Gordon and Breach Science Publishers, New York 1985.

[3] J M. MC Carthy J M. An introduction to theoretical kinematics, London: The MIT Press 1990. 
[4] H. Pottman, and J. Wallner. Computational Line Geometry, Springer-Verlag, Berlin, Heidelberg 2001.

[5] R.A. Abdel-Baky, and R.A Al-Ghefari. On the kinematic geometry of relative screw motions, J. of Mechanical Science and Technology 26 (8) (2012) 2497 2503.

[6] A. Turgut, and H.H Hacısalihoğlu. Timelike ruled surfaces in the Minkowski 3-space. FarEast J. Math. Sci. 5 (1), 83-90 (1997).

[7] N. Hassan, R.A. Abdel-Baky, and F. M. Hamdoon. Ruled surfaces with timelike rulings, Applied Mathematics and Computation 147(1), January (2004), 241-253.

[8] A. Küçük. On the developable timelike trajectory ruled surfaces in Lorentz 3-space $\mathrm{R}_{1}^{3}$ App. Math. and Comp. 157, (2004), 483-489.

[9] M. Onder and H. Huseyin Ugurlu. Frenet frames and invariants of timelike ruled surfaces, Ain Shams Engineering Journal (2013) 4, 507-513.

[10] R. Porteous. Geometric differentiation for the intelligence of Curves and Surfaces, Second edition, Cambridge University Press, Cambridge, 2001.

[11] S. Izumiya and N. Takeuchi, Geometry of ruled surfaces, Applicable Math., in the golden age, (2003), 305-338.

[12] S. Izumiya and N. Takeuchi. New Special Curves and Developable Surfaces, Turk J Math, 28 (2004), 153-163.

[13] I. Shyuichi and W. Yongqiao. Duals of timelike Sabban curves in de Sitter n-space, J. Geom. (2018) 109:31. 